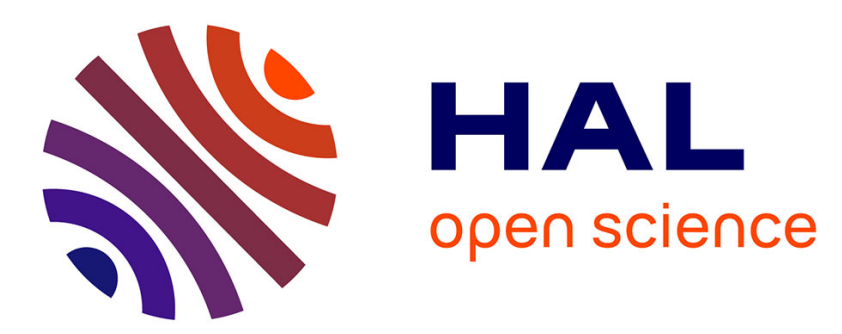

\title{
Evaluation of Level Set reinitialization algorithms for phase change simulation on unstructured grids
}

Guillaume Sahut, Giovanni Ghigliotti, Philippe Marty, Guillaume Balarac

\section{To cite this version:}

Guillaume Sahut, Giovanni Ghigliotti, Philippe Marty, Guillaume Balarac. Evaluation of Level Set reinitialization algorithms for phase change simulation on unstructured grids. 2019. hal-02324919

\section{HAL Id: hal-02324919 \\ https://hal.science/hal-02324919}

Preprint submitted on 22 Oct 2019

HAL is a multi-disciplinary open access archive for the deposit and dissemination of scientific research documents, whether they are published or not. The documents may come from teaching and research institutions in France or abroad, or from public or private research centers.
L'archive ouverte pluridisciplinaire HAL, est destinée au dépôt et à la diffusion de documents scientifiques de niveau recherche, publiés ou non, émanant des établissements d'enseignement et de recherche français ou étrangers, des laboratoires publics ou privés. 


\title{
EVALUATION D’ALGORITHMES DE REINITIALISATION DE LA LEVEL SET POUR LA SIMULATION DU CHANGEMENT DE PHASE SUR MAILLAGES NON STRUCTURES
}

\author{
Guillaume Sahut $^{(1)}$, Giovanni Ghigliotti ${ }^{(1)}$ \\ Philippe Marty ${ }^{(1)}$, Guillaume Balarac ${ }^{(1)}$ \\ ${ }^{(1)}$ Univ. Grenoble Alpes, CNRS, Grenoble INP, LEGI, 38000 Grenoble, France - e-mail: guillaume.sahut @ univ-grenoble-alpes.fr
}

\begin{abstract}
Dans cette étude, on présente différentes méthodes numériques pour la simulation du changement de phase liquide-vapeur (ébullition). On utilise un formalisme Level Set pour capturer l'interface liquide-vapeur. Un tel formalisme nécessite une étape de réinitialisation de la fonction Level Set après advection. Cette étape est critique pour la simulation du changement de phase car elle ne doit ni déplacer l'interface, ni introduire de déformations dans le profil de la fonction Level Set, sous peine de détériorer la précision du calcul de la normale à l'interface et de sa courbure, nécessaires pour définir respectivement la vitesse de l'interface due au changement de phase et le saut de pression à l'interface. On présente d'abord les équations résolues et le couplage des équations de NavierStokes avec le taux de transfert de masse modélisant le changement de phase. Puis on détaille différents algorithmes de réinitialisation de la Level Set pour la simulation numérique de l'ébullition, sur maillages structurés et non structurés. Ces méthodes sont ensuite validées par un cas-test de croissance de bulle statique à taux de transfert de masse fixé. En particulier, on observe qu'à l'instant correspondant au doublement du rayon de la bulle, ce dernier converge en maillages pour toutes les méthodes présentées. Le test concluant sur maillages non structurés ouvre la voie à la simulation du changement de phase liquide-vapeur dans des géométries complexes.
\end{abstract}

MOTS CLEFS : écoulements diphasiques, changement de phase, maillages non structurés, Level Set, ébullition

\section{Evaluation of Level Set reinitialization algorithms for phase change simulation on unstructured grids}

\begin{abstract}
In this study, we present different numerical methods for the simulation of liquid-vapor phase change (boiling). We use a Level Set formalism to capture the liquid-vapor interface. Such a formalism requires a reinitialization (aka redistancing) step of the Level Set function after advection. This step is critical for phase change simulation as it must neither move the interface nor induce perturbations in the Level Set function, otherwise the normal vector to the interface and its curvature, two quantities that are crucial to define respectively the interface velocity due to phase change and the pressure jump at the interface, would be in turn too much perturbed. Here we present a comparison of different reinitialization algorithms of the Level Set function for boiling simulation, on structured and unstructured grids. These methods are then validated against the analytical case of a static growing bubble with a fixed mass transfer rate. In particular, we observe that at the time corresponding to a doubled bubble radius, the error on the bubble radius decreases with the grid cell size for all presented methods.
\end{abstract}

KEYWORDS: two-phase flows, phase change, unstructured grids, Level Set, boiling

\section{INTRODUCTION}

Two-phase flows are encountered in a wide range of industrial applications such as heat exchangers, nucleate boiling or spray cooling. The particularity of two-phase flows is the existence of an interface between the two phases. Numerical simulations of two-phase flows require the localization of the interface. Two-phase flow simulations including liquid-vapor phase change are even more challenging, as the motion of the liquid-vapor interface depends also on the mass transfer rate. There exist several numerical methods to keep track of the interface. In this study we describe different versions of the Level Set method [Osher and Sethian, 1988] on both structured and unstructured grids. These methods are then validated and compared on the analytical case of a static growing bubble with an imposed mass transfer rate. 


\section{GOVERNING EQUATIONS}

We solve the incompressible Navier-Stokes equations

$$
\frac{\partial \vec{u}}{\partial t}+(\vec{u} \cdot \vec{\nabla}) \vec{u}=-\frac{\vec{\nabla} p}{\rho}+\frac{1}{\rho} \vec{\nabla} \cdot \Sigma,
$$

where $\vec{u}$ is the velocity, $p$ the pressure, $\rho$ the density and $\Sigma$ the viscous stress tensor of the given phase. The discontinuity in the velocity field at the interface is given by

$$
[\vec{u}]_{\Gamma}=-\dot{m}\left[\frac{1}{\rho}\right]_{\Gamma} \vec{n},
$$

where $[q]_{\Gamma}=q_{\text {liq }}-q_{\text {vap }}$ denotes the discontinuity, or jump, of the quantity $q$ across the interface $\Gamma, \dot{m}$ is the mass transfer rate (in $\mathrm{kg} \mathrm{m}^{-2} \mathrm{~s}^{-1}$ ) responsible for phase change, and $\vec{n}$ is the interface normal vector pointing towards the liquid phase. When solving (1) we also have to take into account the pressure jump at the interface given by

$$
[p]_{\Gamma}=\sigma \kappa-\dot{m}^{2}\left[\frac{1}{\rho}\right]_{\Gamma},
$$

where $\sigma$ is the surface tension and $\kappa$ the curvature of the interface. Both jumps (2) and (3) are used in the projection method to solve (1) [Tanguy et al., 2014].

In this study, as we focus on the different Level Set approaches available to accurately capture the interface, we assume that the mass transfer rate is fixed. These methods have been implemented in the YALES2 solver [Moureau et al., 2011] and are presented in the next section.

\section{LEVEL SET METHODS ON STRUCTURED GRIDS}

The most challenging task in two-phase flow simulations is the accurate localization and advection of the interface at every time step. Perturbations in interface localization result in a loss of precision and, in the more severe cases, in physical aberrations. With phase change, this well-known problem is even more restrictive. There are several methods to represent the interface. In this work, the Level Set method is used. A Level Set function is a function seen as a set of iso-levels. The liquid-vapor interface is identified as one specific isolevel [Osher and Sethian, 1988]. The Level Set method has two major advantages. First, the advection of the Level Set function in all the computational domain enables the implicit capture of the interface. Second, geometrical properties such as normal vector and curvature of the interface are embedded in the Level Set field. The main drawback of the Level Set method is the mandatory need of a reinitialization step of the Level Set function.

\section{III.1 Case 1 - Signed Distance Function reinitialized by a Hamilton-Jacobi equation}

We start our investigations by the method described by Tanguy et al. [2014]. In this method, the Level Set function is the Signed Distance Function $\phi$ to the interface $\Gamma$ defined for any $\vec{x}$ in the computational domain $\Omega$ by

$$
\phi(\vec{x})=\min _{\vec{x}_{\Gamma} \in \Gamma} \pm\left\|\vec{x}-\vec{x}_{\Gamma}\right\| .
$$

In this case, the interface is identified as the 0 iso-level of the Level Set function [Osher and Sethian, 1988]. The Signed Distance Function is advected by solving the standard advection equation

$$
\frac{\partial \phi}{\partial t}+u_{v a p} \cdot \vec{\nabla} \phi=-\frac{\dot{m}}{\rho_{\text {vap }}}
$$

where $u_{v a p}$ is the vapor phase velocity, $\rho_{v a p}$ is the vapor density and the source term on the right hand side is due to phase change. After advection, the function $\phi$ is reinitialized as a Signed Distance Function by solving the Hamilton-Jacobi PDE

$$
\frac{\partial \phi}{\partial \tau}+S\left(\phi^{0}\right)(\|\vec{\nabla} \phi\|-1)=0
$$


where $\tau$ is a pseudo-time, $S$ is a smoothed sign function defined by Sussman et al. [1994], and $\phi^{0}$ is the previously advected Level Set that needs to be reinitialized. The equation (6) is solved in pseudo-time until convergence, i.e. until $\|\vec{\nabla} \phi\|=1$, which is part of the definition of the Signed Distance Function. The normal vector to the interface $\vec{n}$ and the curvature of the interface $\kappa$ are then given by

$$
\vec{n}=\frac{\vec{\nabla} \phi}{\|\vec{\nabla} \phi\|} \text { and } \kappa=-\vec{\nabla} \cdot \vec{n} .
$$

Note that high precision is needed in the reinitialization of $\phi$ to avoid perturbations in the normal vector and curvature of the interface. To this purpose, the term $\vec{\nabla} \phi$ in (6) is computed using a high-order scheme such as the Fifth Order WENO scheme [Jiang and Peng, 2000]. High-order schemes require higher computational cost, are difficult to implement on unstructured grids and may reduce performance in a parallel code.

\section{III.2 Case 2 - Signed Distance Function reinitialized by the Fast Marching Method}

Another method for the reinitialization of the Signed Distance Function is the Fast Marching Method [Sethian, 1996]. The Fast Marching Method, based on the solution of an Eikonal equation, can be seen as the stationary version of the Hamilton-Jacobi equation (6) and is given by

$$
\|\vec{\nabla} \phi\|=1 .
$$

The solution of equation (8) is based on the propagation of the Signed Distance Function values from the interface along the normal direction to the interface. To limit the computational time needed to perform the Fast Marching Method, we solve it only on a narrow band around the interface, large enough to be able to compute the normal vector and the curvature by equations (7).

\section{III.3 Case 3 - Conservative Level Set on structured grids}

In order to improve mass conservation in each phase, Olsson and Kreiss [2005] proposed the Conservative Level Set method. In this method, the Level Set function $\psi$ is a smeared-out Heaviside function defined for $\vec{x} \in \Omega$ by

$$
\psi(\vec{x})=\frac{1}{2}+\frac{1}{2} \tanh \left(\frac{\phi(\vec{x})}{2 \varepsilon(\vec{x})}\right),
$$

where $\phi$ is the Signed Distance Function and $\varepsilon$ is a scale parameter roughly of the order of the grid cell size. In this case, the interface is identified as the 0.5 iso-level of the Level Set function. The Conservative Level Set is advected by solving the conservative form of the standard advection equation with source term

$$
\frac{\partial \psi}{\partial t}+\vec{\nabla} \cdot\left(\psi \vec{u}_{v a p}\right)=-\vec{u}_{P C} \cdot \vec{\nabla} \psi-\psi \vec{\nabla} \cdot \vec{u}_{\text {vap }},
$$

where the source term is composed of the phase change contribution and the divergence of the vapor velocity, which should be null, but it is recommended to account for this correction to decrease the sensitivity to errors in the evaluation of the velocity field. In (10), the interface velocity due to phase change $\vec{u}_{P C}$ is given by $\vec{u}_{P C}=\frac{\dot{m}}{\rho_{\text {vap }}} \vec{n}$, and the gradient of the Level Set $\vec{\nabla} \psi$ is computed as

$$
\vec{\nabla} \psi(\vec{x})=\frac{1}{4 \varepsilon(\vec{x}) \cosh ^{2}\left(\frac{\phi(\vec{x})}{2 \varepsilon(\vec{x})}\right)} \vec{n},
$$

where $\phi$ is again the Signed Distance Function. This technique to compute $\vec{\nabla} \psi$ is reused from the reinitialization step suggested by Chiodi and Desjardins [2017] and presented below. The Conservative Level Set embeds the interesting property of volume conservation. The normal vector and the curvature of the interface are given by a similar approach as in (7). 
The reinitialization equation for the Conservative Level Set proposed by Olsson and Kreiss [2005] is

$$
\frac{\partial \psi}{\partial \tau}+\vec{\nabla} \cdot(\psi(1-\psi) \vec{n})=\vec{\nabla} \cdot(\varepsilon(\vec{\nabla} \psi \cdot \vec{n}) \vec{n})
$$

where the normal vector is given by

$$
\vec{n}=\frac{\vec{\nabla} \psi}{\|\vec{\nabla} \psi\|} .
$$

Nevertheless, the necessity to set $\varepsilon$ to a small value to improve volume conservation produces sharp gradients in $\psi$ and thus potential oscillations in $\vec{n}$. To avoid this problem, and with the aim of increasing accuracy, Chiodi and Desjardins [2017] built a new reinitialization equation for the Conservative Level Set given by

$$
\frac{\partial \psi}{\partial \tau}=\vec{\nabla} \cdot\left(\frac{1}{4 \cosh ^{2}\left(\frac{\phi_{\text {map }}}{2 \varepsilon}\right)}\left(\left|\vec{\nabla} \phi_{\text {map }} \cdot \vec{n}\right|-1\right) \vec{n}\right),
$$

where the inverse of the Conservative Level Set $\phi_{\text {map }}$ is given by

$$
\phi_{\text {map }}=\varepsilon \log \left(\frac{\psi}{1-\psi}\right) .
$$

The normal vector is computed based on the Signed Distance Function, derived from the Conservative Level Set function by using the Fast Marching Method:

$$
\vec{n}=\frac{\vec{\nabla} \phi_{F M M}}{\left\|\vec{\nabla} \phi_{F M M}\right\|},
$$

where $\phi_{F M M}$ is the Signed Distance Function given by the Fast Marching Method using boundary conditions on the closest nodes to the interface.

\section{LEVEL SET METHODS ON UNSTRUCTURED GRIDS}

\section{IV.1 Case 4 - Signed Distance Function on unstructured grids}

To be able to address complex geometries, algorithms are now extended on unstructured grids. The first unstructured case is similar to Case 1. Only the reinitialization step of the Signed Distance Function is replaced by the method developed on unstructured grids by Dapogny and Frey [2012]. We make use of the MshDist library implementing this method.

\section{IV.2 Case 5 - Conservative Level Set on unstructured grids}

Here we want to extend the methodology of Chiodi and Desjardins [2017] described for structured grids in Section III.3 to unstructured grids. The only difference is the replacement of $\phi_{F M M}$ by the Signed Distance Function given by the method proposed by Dapogny and Frey [2012].

\section{NUMERICAL RESULTS}

We validate the different Level Set reinitialization methods presented in the previous section on the case of a 2D static growing bubble with a fixed mass transfer rate from [Tanguy et al., 2014]. The initial bubble radius is $R_{0}=10^{-3} \mathrm{~m}$ and the imposed mass transfer rate is $\dot{m}=10^{-1} \mathrm{~kg} \mathrm{~m}^{-2} \mathrm{~s}^{-1}$. The simulations are performed until final time $t_{f}=10^{-2} \mathrm{~s}$ needed for the bubble radius to double the initial radius. The other physical parameters of interest are $\rho_{\text {liq }}=10^{3} \mathrm{~kg} \mathrm{~m}^{-3}, \rho_{\text {vap }}=1 \mathrm{~kg} \mathrm{~m}^{-3}, \sigma=7 \times 10^{-2} \mathrm{~N} \mathrm{~m}^{-1}, \mu_{\text {liq }}=10^{-3} \mathrm{~kg} \mathrm{~m}^{-1} \mathrm{~s}^{-1}$ and $\mu_{\text {vap }}=1.78$ $\times 10^{-5} \mathrm{~kg} \mathrm{~m}^{-1} \mathrm{~s}^{-1}$. In Table 1 , the methods used in all cases are summarized. 


\begin{tabular}{|c|c|c|c|c|c|}
\hline Grid topology & \multicolumn{2}{|c|}{ Structured } & \multicolumn{2}{c|}{ Unstructured } \\
\hline Level Set type & \multicolumn{2}{|c|}{ SDF } & CLS & SDF & CLS \\
\hline $\begin{array}{c}\text { Reinitialization } \\
\text { method }\end{array}$ & HJ eq. & FMM & $\begin{array}{c}\text { [Chiodi and } \\
\text { Desjardins, 2017] }\end{array}$ & $\begin{array}{c}\text { [Dapogny and } \\
\text { Frey, 2012] }\end{array}$ & $\begin{array}{c}\text { Frey, 2012] + } \\
\text { [Chiodi and } \\
\text { Desjardins, 2017] }\end{array}$ \\
\hline Cases & Case 1 & Case 2 & Case 3 & Case 4 & Case 5 \\
\hline
\end{tabular}

Table 1. Summary of all cases presented in the previous sections. SDF stands for Signed Distance Function, CLS for Conservative Level Set, HJ for Hamilton-Jacobi and FMM for Fast Marching Method.

The five cases have been computed on four different grid cell sizes. As an example, Fig. 1 shows the results of Case 5 at final time on four different unstructured grids. The relative error on the bubble radius with respect to the theoretical radius at final time is given for all cases at final time in Fig. 2 (a). One can see that on the finest grid, the Case 5 detailed in Fig. 1 presents the highest relative error. The five methods have a convergence rate close to one. For the finest grid, all relative errors on the bubble radius are below $1 \%$. The relative errors on the normal vector and the curvature are shown in respectively Fig. 2 (b) and 2 (c). The results show that the error on the normal vector decreases at order 1 for all methods including the Signed Distance Function on unstructured grids (Case 4). For Case 5, i.e. for the Conservative Level Set on unstructured grids, further work is needed to improve the convergence of both normal vector and curvature.

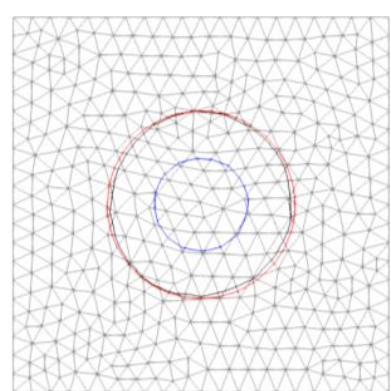

(a)
0.1

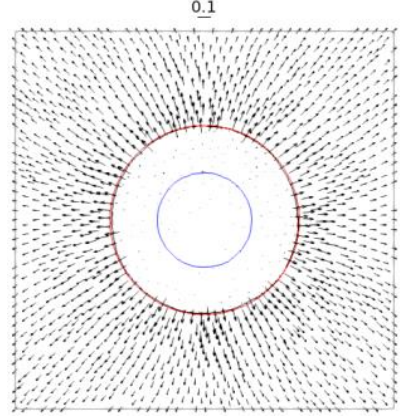

(b)

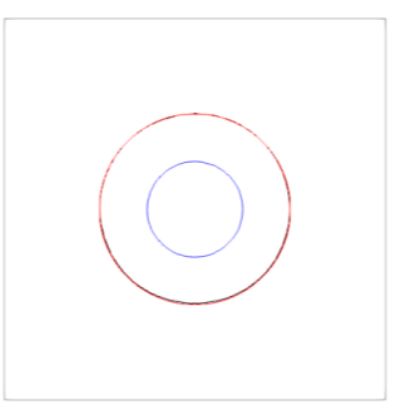

(c)

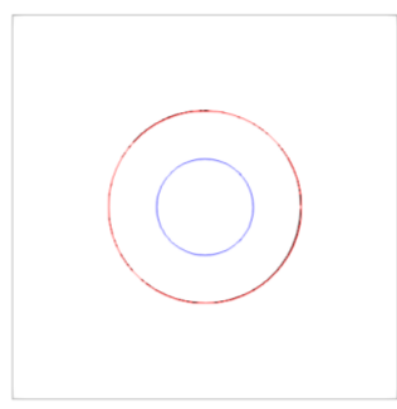

(d)

Fig. 1. The results for the Case 5 on four different unstructured grids are plotted at final time with a characteristic cell size of (a) $4 \times 10^{-4} \mathrm{~m}$, (b) $2 \times 10^{-4} \mathrm{~m}$, (c) $1 \times 10^{-4} \mathrm{~m}$ and (d) $5 \times 10^{-5} \mathrm{~m}$. The initial interface is plotted in blue, the computed interface in black, and the theoretical interface in red. For clarity, only the coarsest grid is represented in (a). The computed liquid and vapor velocity fields are plotted in (b).

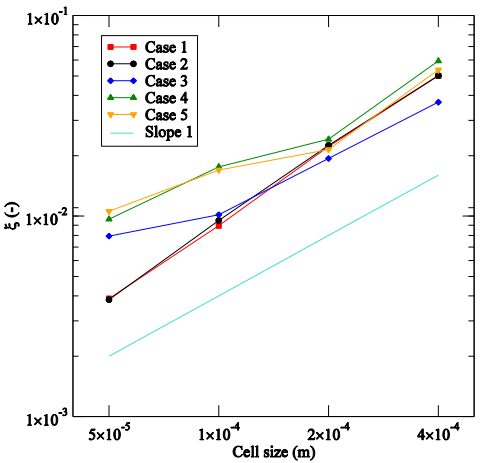

(a)

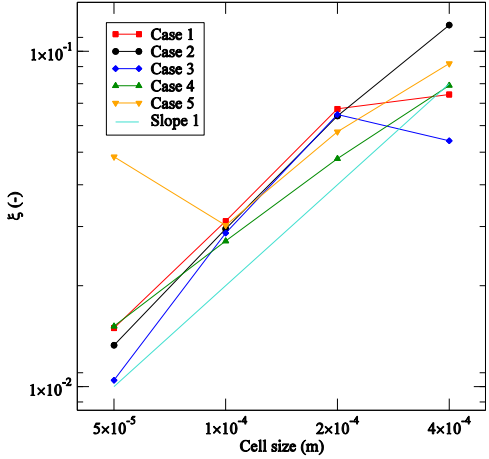

(b)

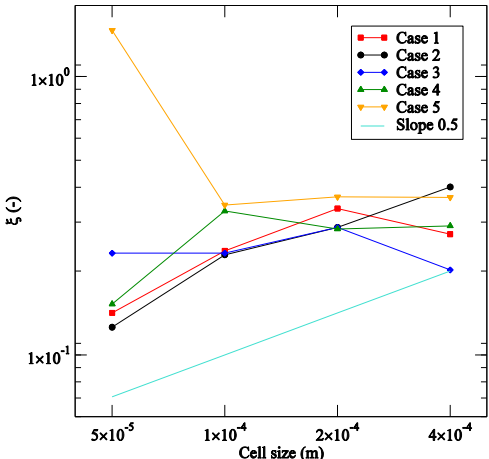

(c)

Fig 2. The normalized $\mathrm{L}^{\infty}$ norm of the error on the bubble radius $\xi$ is plotted at final time for the five cases on four different grid cell sizes (a). The analogous error for the normal vector (b) and for the curvature (c). 


\section{CONCLUSION AND PERSPECTIVES}

In this study, several numerical methods for the advection and reinitialization of the Level Set function have been presented in the context of liquid-vapor phase change. These methods have been validated and compared on the case of a static growing bubble with a fixed mass transfer rate. All methods present a convergence rate with the grid cell size close to one. Two of the five methods presented are designed for unstructured grids. The ability to accurately compute the interface position allows the quantitative simulation of liquid-vapor phase change on unstructured grids. This opens the path to numerical simulations of liquid-vapor phase change on complex geometries.

The main perspective of our work is the simulation of phase change on unstructured grids with a computed mass transfer rate which depends on the thermal fluxes at the interface and on the latent heat of the fluid.

\section{ACKNOWLEDGMENTS}

This work has been supported by the LabEx Tec21 (Investissements d'Avenir-Grant Agreement No. ANR11-LABX-0030). Vincent Moureau and Ghislain Lartigue from the CORIA lab and the SUCCESS scientific group are acknowledged for providing the YALES2 code. G. B. is also grateful for the support from Institut Universitaire de France. The authors would like to thank Patrick Begou for support on algorithms implementation and Charles Dapogny for support on the MshDist library.

\section{REFERENCES}

Chiodi R., Desjardins O. (2017). - A reformulation of the conservative level set reinitialization equation for accurate and robust simulation of complex multiphase flows. J. Comput. Phys., 343: 186-200.

Dapogny C., Frey P. (2012). - Computation of the signed distance function to a discrete contour on adapted triangulation. Calcolo, 49(3): 193-219.

Jiang G.-S., Peng D. (2000). - Weighted ENO Schemes for Hamilton-Jacobi Equations. SIAM J. Sci. Comput., 21(6): 2126-2143.

Moureau V., Domingo P., Vervisch L. (2011). — Design of a massively parallel CFD code for complex geometries. CR Mec, 339(2-3): 141-148.

Olsson E., Kreiss G. (2005). - A conservative level set method for two phase flow. J. Comput. Phys., 210: 225-246.

Osher S., Sethian J. A. (1988). — Fronts propagating with curvature-dependent speed: Algorithms based on Hamilton-Jacobi formulations. J. Comput. Phys., 79: 12-49.

Sethian J. A. (1996). — A Fast Marching Level Set Method for Monotonically Advancing Fronts. Proc. Natl. Acad. Sci., 93(4): 1591-1595.

Sussman M., Smereka P., Osher S. (1994). - A Level Set Approach for Computing Solutions to Incompressible Two-Phase Flow. J. Comput. Phys., 114: 146-159.

Tanguy S., Sagan M., Lalanne B., Couderc F., Colin C. (2014). - Benchmarks and numerical methods for the simulation of boiling flows. J. Comput. Phys., 264: 1-22. 